Weed Science 2017 65:1-3

(C) Weed Science Society of America, 2017

\title{
Editorial for Weed Science, Volume 65
}

\author{
William K. Vencill*
}

This has been an exciting year for Weed Science. For the past year, we have been transitioning from Allen Press to Cambridge University Press. This will be our first issue with Cambridge University Press. The journal will go from four to six issues per year and will be online only. One of the benefits of being online only will be that we no longer will charge for color photographs or figures, so I would encourage authors to use more color in their submissions. The manuscript submission process should be seamless for authors, as we are still using the underlying software that was present in PeerTrack, it will now just be referred to as Editorial Manager. One last change that authors will see will be in early-view articles. Instead of the submitted manuscript being posted online after acceptance, Weed Science will post the completed article (known as the Version of Record) in the First View section. This will be the final article minus the page numbers.

Weed Science continues to be one of the premier journals in the discipline and in the agronomic sciences. The editorial board and the staff strive to improve the efficiency of the publication process and improve the scope of the journal. Weed Science seeks to publish more reviews and symposium articles.

\section{Editorial Board 2016}

Muthukumar V. Bagavathiannan

Carlene Chase

Bhagirath Chauhan

Sharon Clay

Adam Davis

Franck E. Dayan

J. Anita Dille

Timothy L. Grey

Marie Jasieniuk

Ramon Leon

John L. Lindquist

Vijay Nandula

Chris Preston

DOI: $10.1017 /$ wsc.2016.19

*Professor and Editor, Department of Crop and Soil Sciences, University of Georgia, Athens, GA 30602. Corresponding author's email: wvencill@uga.edu
This past year, Weed Science published a special issue of Weed Science from presenters at the WSSA Herbicide Resistance Summit II.

For the 12 months preceding September 1, 2016, 205 manuscripts were submitted to Weed Science compared with 211 in the previous 12-month period. Of the manuscripts accepted, $45.4 \%$ were accepted compared with $44.3 \%$ the previous year. The time from submission to first decision was 37 days this past year compared with 44 days for the previous year.

The impact factor for Weed Science increased from 1.870 to 1.993 this past reporting cycle, while the 5 year impact factor increased from 1.928 to 2.166 . I believe the latest impact factor and the 5 year impact factor are the best the journal has ever had.

For the year preceding September 1, 2016, the journal depended on 276 unique reviewers from 28 countries for peer review. The excellent reputation of the journal would not be possible without the cutting-edge research submitted by authors, the diligent work of the editorial board, and the hundreds of referred reviews that are submitted annually. I wish to thank the members of the editorial board and reviewers for 2016.

Dean Riechers

Hilary Sandler

Steven Seefeldt

Patrick J. Tranel

Martin M. Williams II

Weed Science Reviewers 2016

Ricardo Alcántara-de la Cruz

James V. Anderson

Randy L. Anderson

Lars W. J. Anderson

Gregory R. Armel

Antonia D. Asencio

Albert O. Ayeni

Matt Anthony Bahm

Sanjeev K. Bangarwa

Travis Bean

Laura Louise Beaton

Hugh J. Beckie 
Roberto Benech-Arnold

Lori K. Benoit

Thierry Eric Besancon

Carmen K. Blubaugh

Rick A. Boydston

Daniel Brainard

James T. Brosnan

Nilda R. Burgos

Ian C. Burke

John Cardina

Andrea Cavalieri

Aman Chandi

Rakesh S. Chandran

Carlene A. Chase

Sushila Chaudhari

William J. Chism

Michael J. Christoffers

Warren Conway

Roberto J. Crespo

Daniel Chad Cummings

Mark Andrew Czarnota

Caleb D. Dalley

Henri Darmency

Richard Davis

Christophe Delye

Jeffrey F. Derr

Joseph M. DiTomaso

Jonathan Castel Djietror

David Lee Dornbos Jr.

Ryan James Edwards

Ilias George Eleftherohorinos

Stephen F. Enloe

Jonathan E. Ephrath

Amber Eytcheson

Jason Ferrell

Lloyd Flack

Michael Luke Flessner

Silvia Fogliatto

Frank Forcella

Chaim Frankel

George Frisvold

Todd A. Gaines

Travis W. Gannon

David Gealy

Roland Gerhards

Darci Giacomini

Kevin D. Gibson

Gurjeet S. Gill

Rakesh Kumar Godara

Greta Gramig

Robert H. Gulden

Dustin Haines

Bradley D. Hanson
Stuart Patrick Hardegree

K. Neil Harker

Nick T. Harre

Kent Kent Harrison

Abul Hashem

Erin C. Hill

Melissa J. Hills

David P. Horvath

Lynn Ingegneri

Jordi Izquierdo

Marie A. Jasieniuk

Prashant Jha

Amit J. Jhala

Nicholas Royal Jordan

Mithila Jugulam

Miroslav Jursík

Yalcin Kaya

Jason P. Kelley

James J. Kells

Ken Klemow

Susan Koehler

Nicholas E. Korres

Robert J. Kremer

Greg Kruger

Vipan Kumar

Sarah Lancaster

D. K. Lee

Ramon G. Leon

Lingxu Li

Matt Liebman

Rick S. Llewellyn

Mark M. Loux

Drew J. Lyon

Rong $\mathrm{Ma}$

Mayank S. Malik

Hiroshi Matsumoto

Jeff Maughan

William B. McCloskey

James D. McCurdy

Joseph Scott McElroy

Kristen E. McNaughton

Fabian Daniel Menalled

Timothy W. Miller

John Miranowski

Steven B. Mirsky

Patrick Moran

Thomas C. Mueller

Hanwen Ni

Robert Edward Nurse

Dennis Calvin Odero

David Olszyk

Maria Dolores Osuna Ruiz

Bassiaka Ouatara

2 - Weed Science 65, January-February 2017 
Daniel Owens

William Louis Patzoldt

Ed Peachey

Alejandro Perez-Jones

Abelino Pitty

Angela R. Post

Christopher Preston

Andrew Jennings Price

Neha Rana

Sandeep Singh Rana

Jordi Recasens

Dawn Refsell

Emilie E. Regnier

Kurt Reinhart

Karen A. Renner

Dilpreet Riar

Daniela Neves Ribeiro

Darren E. Robinson

Diego Rubiales

Francesc Xavier Sans

Laura Scarabel

Jill Schroeder

Brian J. Schutte

Julio Alejandro Scursoni

Dale L. Shaner

Vinod K. Shivrain

Sudeep Sidhu

Peter H. Sikkema

Samunder Singh

Vijay Singh

Reid J. Smeda

Richard G. Smith

Lynn M. Sosnoskie

Josef Soukup
Christy L. Sprague

Daniel O. Stephenson IV

Tracy M. Sterling

David E. Stoltenberg

Jens Carl Streibig

Clarence J. Swanton

François J. Tardif

Parsa Tehranchian

Joel Torra Farré

Hilmi Torun

Ilias S. Travlos

Te-Ming Paul Tseng

Esa Tyystjärvi

Mark VanGessel

Michael Walsh

Guang-Xi Wang

JinXin Wang

Sarah Ward

Rodrigo Werle

Paula Westerman

James H. Westwood

Joe Yenish

Melinda Yerka

Carla Yerkes

Bryan G. Young

Stephen L. Young

Rafael Zas

Mingqi Zheng

Richard Zollinger

\section{Reference}

2015. Journal Citation Reports Science Edition. 2016. New York: Thomson Reuters. 